\begin{tabular}{|c|c|}
\hline Title & $\begin{array}{l}\text { Biological effect of dose distortion by fiducial markers in spot-scanning proton therapy with a limited number of fields: } \\
\text { A simulation study }\end{array}$ \\
\hline Author(s) & $\begin{array}{l}\text { Matsuura, Taeko; Maeda, Kenichiro; Sutherland, Kenneth; Takay anagi, Taisuke; Shimizu, Shinichi; Takao, Seishin; } \\
\text { Miyamoto, Naoki; Nihongi, Hideaki; T oramatsu, Chie; Nagamine, Y oshihiko; Fujimoto, Rintaro; Suzuki, Ryusuke; } \\
\text { Ishikawa, Masay ori; U megaki, Kikuo; Shirato, Hiroki }\end{array}$ \\
\hline Citation & $\begin{array}{l}\text { Medical Physics, 39(9), } 55845591 \\
\text { https://doi.org/10.1118/1.4745558 }\end{array}$ \\
\hline Issue Date & $2012-09$ \\
\hline Doc URL & http:/hdl.handle.net/2115/50429 \\
\hline Type & article (author version) \\
\hline File Information & MP39-9_5584-5591.pdf \\
\hline
\end{tabular}

Instructions for use 


\section{Biological effect of dose distortion by fiducial markers in spot-scanning proton therapy with a limited number of fields: a simulation study}

Running head: Biological effect of proton dose-distortion by fiducials

Taeko Matsuura ${ }^{1}$, Kenichiro Maeda ${ }^{1}$, Kenneth Sutherland ${ }^{1}$, Taisuke Takayanagi ${ }^{2}$, Shinichi Shimizu ${ }^{2}$, Seishin Takao ${ }^{1}$, Naoki Miyamoto ${ }^{1}$, Hideaki Nihongi ${ }^{1}$, Chie Toramatsu ${ }^{1}$, Yoshihiko Nagamine ${ }^{4}$, Rintaro Fujimoto $^{3}$, Ryusuke Suzuki ${ }^{1}$, Masayori Ishikawa ${ }^{1}$, Kikuo Umegaki $^{2}$, Hiroki Shirato ${ }^{2}$

${ }^{1}$ Department of Medical Physics, Hokkaido University Graduate School of Medicine

${ }^{2}$ Hitachi, Ltd., Hitachi Research Laboratory

${ }^{3}$ Department of Radiation Medicine, Hokkaido University Graduate School of Medicine

${ }^{4}$ Hitachi, Ltd., Hitachi Works

Address correspondence to: Taeko Matsuura PhD

Graduate School of Medicine, Hokkaido University, North 15, West 7, Kita-ku, Sapporo, Hokkaido, 060-8638, Japan

Tel.: +81- 11 - 706- 5254, Fax: +81- 11 -706 -5254

E-mail: matsuura@med.hokudai.ac.jp 


\begin{abstract}
Purpose: In accurate proton spot-scanning therapy, continuous target tracking by fluoroscopic X-ray during irradiation is beneficial not only for respiratory moving tumors of lung and liver but also for relatively stationary tumors of prostate. Implanted gold markers have been used with great effect for positioning the target volume by a fluoroscopy, especially for the cases of liver and prostate with the targets surrounded by water-equivalent tissues. However, recent studies have revealed that gold markers can cause a significant underdose in proton therapy. This paper focuses on prostate cancer and explores the possibility that multiple-field irradiation improves the underdose effect by markers on Tumor Control Probability (TCP).

Methods: A Monte Carlo simulation was performed to evaluate the dose distortion effect. A spherical gold marker was placed at several characteristic points in a water phantom. Markers were with two different diameters of $2 \mathrm{~mm}$ and $1.5 \mathrm{~mm}$, both visible on fluoroscopy. Three beam arrangements of SFUD (single-field uniform dose) were examined: one lateral field, two opposite lateral fields, and three fields (two opposite lateral fields + anterior field). The Relative Biological Effectiveness (RBE) was set to 1.1 and a dose of 74 Gy (RBE) was delivered to the target of a typical prostate size in 37 fractions. The ratios of TCP to that without the marker $\left(\mathrm{TCP}_{\mathrm{r}}\right)$ were compared with the parameters of the marker sizes, number of fields, and marker positions. To take into account the dependence of biological parameters in TCP model, $\alpha / \beta$ values of $1.5,3$, and 10 Gy (RBE) were considered.
\end{abstract}

Results: It was found that the marker of $1.5 \mathrm{~mm}$ diameter does not affect the TCPs with all $\alpha / \beta$ values when two or more fields are used. On the other hand, if the marker size is $\phi 2 \mathrm{~mm}$, more than two irradiation fields are required to suppress the decrease in TCP from $\mathrm{TCP}_{\mathrm{r}}$ by less than $3 \%$. This is especially true when multiple (two or three) markers are used for alignment of a patient.

Conclusions: It is recommended that $\phi 1.5 \mathrm{~mm}$ markers be used to avoid the reduction of TCP as well as to spare the surrounding critical organs, as long as the markers are visible on X-ray fluoroscopy. When $\phi 2 \mathrm{~mm}$ markers are implanted, more than two fields should be used and the markers should not be placed close to the distal edge of any of the beams.

Key Words: Proton spot-scanning therapy, Tumor tracking, Fiducial marker, Prostate cancer, Tumor Control Probability 


\section{INTRODUCTION}

The number of proton therapy centers which adopt the spot-scanning technique has been increasing worldwide. Spot-scanning proton therapy has an advantage to achieve high dose conformity by changing the dosage and the position of each pencil beam (spot) individually under computer control. However, at the same time, targeting accuracy within the order of millimeters must be achieved by various image-guided radiotherapy (IGRT) techniques. During irradiation, continuous target tracking by a fluoroscopic X-ray system is useful for respiratory moving tumors ${ }^{1}$. It has been reported that even the prostate can undergo more than $10 \mathrm{~mm}$ of intra-fractional motion during 1 minute ${ }^{2}$, which suggests that real-time tracking is also beneficial for the prostate ${ }^{3}$. Due to their high opacity, gold markers have been used with great effect especially for the cases of liver and prostate with the targets surrounded by water-equivalent tissues. Gold fiducial markers have been in clinical use in proton therapy centers ${ }^{4}$. However, recent studies have revealed that a certain size of cylindrical gold markers can cause as much as $85 \%$ of proton underdose mainly due to their high electron density ${ }^{5,67}$. Several proposals to mitigate dose distortion have been made such as the reduction of marker size ${ }^{7}$, the optimization of marker compounds with carbon-coated ceramic ${ }^{8}$ or mixture of microscopic gold particles and polymers ${ }^{9}$.

An alternate solution may be to diminish the dose distortion effect by increasing the number of fields ${ }^{10}$. Because proton pencil beam scanning technique does not require replacement of the apertures and range compensators field by field, the treatment time will not be increased so much by the use of multiple fields. On the other hand, there is a limit to the number of fields. First of all, the low-dose region is expanded by an increase in the number of fields, which could cancel the advantages of proton therapy. Secondly, for spot-scanning proton therapy, the plan quality tends to be worse as the number of field exceeds a certain threshold when single-field optimization (SFO) is used ${ }^{11}$. This is due to the minimum monitor unit (MU) for delivering each spot, which is $0.005 \mathrm{MU}$ at our institution. Therefore, it is necessary to use a minimum number of fields while at the same time to control the effect of dose distortion due to the presence of gold markers to be within an acceptable range.

In this research, we focus on prostate cancer and explore the possibility that multiple-field irradiation improves the underdose effect by markers on Tumor Control Probability (TCP) using a Monte Carlo simulation. Three beam arrangements of SFUD (single-field uniform dose) were examined: one lateral field, two opposite lateral fields, and three fields (two opposite lateral fields + anterior field). More than three fields were not considered 
because the MU constraint limits the number of fields to three for 2 Gy (RBE) irradiation to the target of a typical prostate size with $8 \mathrm{~mm}$ spot spacing. We also investigated two different sizes of gold marker (2 $\mathrm{mm}$ and $1.5 \mathrm{~mm}$ in diameter), which are both visible on fluoroscopy. They were placed at several positions in the prostate that have different characteristics in terms of proton momentum distribution, which produce different dose distortions. We considered multiple $\alpha / \beta$ values of 1.5, 3, and $10 \mathrm{~Gy}(\mathrm{RBE})^{12,13,14}$ for the evaluation of TCP, because there are still debate on $\alpha / \beta$ value for prostate tumors at this moment. The required number of fields was then determined for each marker size such that the TCP reduction is within an acceptable range for all position of a marker and for any $\alpha / \beta$ values.

\section{MATERIALS AND METHODS}

\section{A. Simulation setup}

Figure 1 shows the water phantom geometry, coordinate system, and beam directions. The phantom size $(38 \times$ $20 \times 20 \mathrm{~cm}^{3}$ ) was determined based on the average anatomical measurements of water equivalent length made from computed tomography scans of 10 prostate cancer patients' pelvises. Clinical target volume (CTV) was defined as prostate and its size ranges from 28 to $74 \mathrm{~cm}^{3}\left(44 \mathrm{~cm}^{3}\right.$ on average $)$ over the 10 patients. In the simulation, it was represented as a cube of an equivalent volume placed at the center of the phantom and the side length was $3-4.2 \mathrm{~cm}$ (3.5 cm on average). PTV margin of $3 \mathrm{~mm}$, which is clinically used in Hokkaido University Hospital for prostate IMRT, was added for each size of CTV.

The coordinate system is defined with respect to the treatment room; the origin is at the isocenter (IC) which coincides with the center of the target. We considered the following three beam directions: right-lateral (gantry angle 270 degrees, field 1), left-lateral (gantry angle 90 degrees, field 2), and anterior (gantry angle 0 degrees, field 3). The proton absorbed-dose distributions were simulated using the Geant4 Monte Carlo code (ver. 4.9.4).

The binary cascade model ${ }^{15}$ was used for the nuclear interactions. The production thresholds for gamma, electrons and positrons are needed to avoid the infrared divergences and they were all set to $0.1 \mathrm{~mm}$. The Hokkaido University spot-scanning beam treatment nozzle as well as the beam characteristics designed by Hitachi (Hitachi Ltd., Japan) was implemented (Figure 2). The spot size depends on the beam energy and it was $3.6 \sim 6.9 \mathrm{~mm}(\sigma)$ at the IC in air. The primary protons were generated just above the vacuum window, which was located about 2.5 $\mathrm{m}$ from the IC. For one spot, we simulated 600,000 primary protons. The dose was scored in cubic volumes of 0.5 
$\mathrm{mm}$ side length in order to obtain statistical uncertainty less than $2 \%$ at the distal border of the PTV.

Spot weight optimization was performed by SFUD. For all fields, the spacing between spots was set to $8 \mathrm{~mm}$. The simulation was made for minimum, average, and maximum size of CTV which are denoted as $\mathrm{CTV}_{\text {min }}$, $\mathrm{CTV}_{\text {ave }}$, and $\mathrm{CTV}_{\max }$, respectively. For $\mathrm{CTV}_{\text {ave }}$, iso-energy layers of 157-180 MeV (11 layers) were used for the lateral beams (fields 1 and 2) and 103-132 MeV (21 layers) for the anterior beam (field 3). Fields 1 and 2 required 891 spots to cover the PTV volume, while field 3 needed 1701 spots. We assumed a dose prescription of 74 Gy $(\mathrm{RBE})$ in 37 fractions to the isocenter in accordance with a previous report ${ }^{16}$. The Relative Biological Effectiveness (RBE) was set to 1.1. When three fields were used, the minimum MU per spot was 0.007 for fields 1 and 2 and 0.005 for field 3 at the lowest iso-energy layer. This indicates that as long as we constrain ourselves to SFUD with $8 \mathrm{~mm}$ spot spacing, the maximum field number is three for $2 \mathrm{~Gy}$ (RBE) irradiation to this target. The calculated dose deviation from the prescribed dose comes from both small ripples within the spread-out Bragg peaks in a treatment plan and statistical uncertainty. It was within $\pm 5 \%$ for more than $99.9 \%$ of the CTV. In a same manner, the dose distribution for $\mathrm{CTV}_{\min }$ and $\mathrm{CTV}_{\max }$ were constructed by using corresponding iso-energy layers.

\section{B. Gold marker placement}

Two sizes of spherical gold marker with diameters of $2 \mathrm{~mm}$ and $1.5 \mathrm{~mm}$ were investigated. As shown in Figure 3, both sizes were recognized by a fluoroscopy when inserted in a pelvic phantom (100 kV, $80 \mathrm{mAs}, 4 \mathrm{msec}(\mathrm{AP})$, $120 \mathrm{kV}, 80 \mathrm{mAs}, 4 \mathrm{msec}(\mathrm{LR}))$. The fluoroscopic images of the $\phi 2 \mathrm{~mm}$ marker were clear, while those of the $\phi 1.5$ mm marker might be not so clear to discern in some patients.

Markers were placed at several characteristic points for each beam arrangement (Fig. 4). In the case of one field, 5 points were considered: the IC (C), the proximal (A) and distal (E) points, and the intermediate points between IC and the proximal point (B) and between IC and the distal point (D). In the case of two and three fields, the representative 3 and 15 points were investigated, respectively. When a marker is placed at an off-central axis position, the dose distortion due to the presence of the marker is assumed to be the parallel shift of that produced when the marker is place at the central axis. This is a good approximation because the SOBP width is a constant for the cubic PTV and because the marker positions are chosen so that they are more than $5 \mathrm{~mm}$ distant from the PTV boundary. The dose distortion that occurs in a cylindrical region of a diameter less than $5 \mathrm{~mm}$, does not 
overlap with the PTV boundary (see Sec. III).

\section{TCP model}

In order to evaluate the biological effect of the dose distortion, TCP values were evaluated from the dose distribution derived by the above-mentioned procedure. First of all, the linear quadratic (LQ) model was used for the calculation of surviving fraction $(\mathrm{SF})$ :

$$
\mathrm{SF}=\mathrm{e}^{-\mathrm{E}(D)},
$$

where

$$
\mathrm{E}(D)=\alpha D+\frac{\beta}{n} D^{2}
$$

Here $\alpha$ and $\beta$ characterize intrinsic radio-sensitivity, $D$ is the total dose that is generally different from point to point in the prostate, $n$ is the number of dose fractions. Then, the Poisson TCP model:

$$
\mathrm{TCP}=\prod_{\text {voxel } i} e^{-n_{i} \mathrm{SF}(D(i))}, n_{i}=\frac{\rho_{c} V}{N_{\text {voxel }}}
$$

was used to link cell killing to bulk clinical effect where $N_{\text {voxel }}$ varies according to the CTV size (from $60^{3}$ to $84^{3}$ in this simulation). $D(i)$ is the local dose at the $i$-th voxel, $\rho_{c}$ is the initial number density of clonogenic cells, $V$ is the CTV volume, and $n_{\mathrm{i}}$ is the cell number in $i$-th voxel. We assume a uniform CTV clonogenic cell density of $\rho_{c}=10^{6}$ $\mathrm{cm}^{-317}$. In the evaluation, the voxels that contain gold markers are excluded.

The biological parameters used in this study are summarized in Table 1. For each parameter set, the TCP curve for homogeneous dose distribution was shown in Figure 5. Three $\alpha / \beta$ values of 1.5, 3, and 10 Gy (RBE) were examined. For a fixed $\alpha / \beta$, parameter $\alpha$ was determined so that the TCP at 74 Gy (RBE) in 37 fractions is $94 \%$ for $\mathrm{CTV}_{\text {ave. }}$ This value corresponds to the clinical data available on proton therapy. In report ${ }^{16}$, it was shown that biochemical relapse-free survival rate (bNED) at 3 years was $94 \%$ for Gleason score $<8$ patient group. The similar percentage is also seen in report ${ }^{18}$ in which bNED at 5 -years was more than $92 \%$ for T1-T2a patients with the same dose prescription. As far as the authors know, additional clinical data with different dose prescriptions and same patient group was not available. With those data, the variability in clonogen sensitivities within a tumor $\left(\sigma_{\text {ind }}\right)$ and/or the variability from patient to patient $\left(\sigma_{\text {pop }}\right)$ would be determined by curve fitting ${ }^{19,20}$. In this study, $\sigma_{\text {ind }}$ and $\sigma_{\mathrm{pop}}$ are set to zero, which may result in an overestimation of TCP reduction by the underdosage ${ }^{20}$. 


\section{Method of evaluation}

The ratios of TCP to that without the marker $\left(\mathrm{TCP}_{\mathrm{r}}\right)$ were compared with the parameters of the marker sizes, number of fields, and marker positions for each $\alpha / \beta$. For each parameter set, the ratio $\mathrm{R}_{\mathrm{TCP}}=\mathrm{TCP} / \mathrm{TCP} \mathrm{r}_{\mathrm{r}}$ was obtained for three CTV volumes and the minimum value of those, namely $\mathrm{R}_{\mathrm{TCP}}^{\mathrm{V}}=\min \left\{\mathrm{R}_{\mathrm{TCP}}\left(\mathrm{CTV}_{\min }\right)\right.$, $\mathrm{R}_{\mathrm{TCP}}\left(\mathrm{CTV}_{\text {ave }}\right), \mathrm{R}_{\mathrm{TCP}}\left(\mathrm{CTV}_{\max }\right)$ \}, were calculated. Here, as can be seen from eq. (3), $\mathrm{TCP}_{\mathrm{r}}$ decreases as the $\mathrm{CTV}$ volume increases and it was $96 \%, 94 \%$, and $90 \%$ for $\mathrm{CTV}_{\min }, \mathrm{CTV}_{\text {ave }}$, and $\mathrm{CTV}_{\max }$, respectively. The tolerance for TCP reduction is defined as $\delta$ TCP $<3 \%$, where $\delta T C P \equiv 100 \times\left(1-\mathrm{R}_{\text {TCP }}^{\mathrm{V}}(\%)\right.$. $\delta$ TCP for multiple markers is approximately the sum of $\delta$ TCP's caused by each marker as long as $\left(1-\mathrm{R}_{\mathrm{TCP}}^{\mathrm{V}}\right) \ll 1$ is satisfied. In order to understand the results, the depth dose distributions, the plane dose distribution along the central beam axis, and the minimum dose in CTV $\left(\mathrm{D}_{\min }\right)$ were shown for $\mathrm{CTV}_{\text {ave }}$.

\section{RESULTS}

\section{A. Dose distribution}

Figure 6 shows the depth dose distribution along the central beam axis for one field with both sizes of gold markers. In each figure, three plots for one lateral field with marker positions (A), (C), and (E) in Figure 4 (a) are shown with the reference (without a marker). Figure 7 shows the plane dose distribution for one field (a), two opposite fields (b), and three fields (c) along the central beam axis for gold markers with $\phi 2 \mathrm{~mm}$ placed at the IC, which are (C), (C'), and (C') in Figure 4, respectively. Both graphs are shown for $\mathrm{CTV}_{\text {ave }}$. The minimum dose is listed in Table 2 for all conditions.

As shown in Fig. 6 and 7, the gold marker trails a thin cold line in the downstream region. Proton fluence is reduced because protons stop in the gold marker or are strongly scattered by gold nuclei, i.e., $\theta_{\mathrm{Au}} \sim 6 \theta_{\mathrm{H} 2 \mathrm{O}}$. Compared with the size of the CTV, however, the cold line thickness and its volume are sufficiently small, that is, within the cylindrical region of $\phi 5 \mathrm{~mm}$. The cold line length is shorter as the marker approaches the distal edge, but at the same time, $\mathrm{D}_{\min }$ is further reduced because larger number of protons stops in the marker as well as the fewer protons are scattered in the path by multiple Coulomb scattering (MCS). The exception is the case when the marker is very close to the CTV boundary (a few mm). In that case, the point where the dose shadowing is maximum is outside of CTV and it does not coincide with $\mathrm{D}_{\min }$. For one field, $\mathrm{D}_{\min }$ is $24.1 \mathrm{~Gy}(\mathrm{RBE})(\phi 2 \mathrm{~mm})$ and 
$39.4 \mathrm{~Gy}(\mathrm{RBE})(\phi 1.5 \mathrm{~mm})$ when the marker is placed rather close to the distal edge ((D) or (E) in Figure 4). This improves to $\mathrm{D}_{\min }=51.4 \mathrm{~Gy}(\mathrm{RBE})(\phi 2 \mathrm{~mm})$ and $57.9 \mathrm{~Gy}(\mathrm{RBE})(\phi 1.5 \mathrm{~mm})$ by using three fields (see Table 2$)$. The minimum dose occurs at a position that is close to the distal edge for either one of the beams.

When a marker is placed upstream of the CTV, the recovery of the dose occurs downstream of the CTV (Fig. 6 (A) and (C)) because the neighboring protons scatter in the cold line. For $\phi 1.5 \mathrm{~mm}$ marker at position (A), the dose recovers to almost $90 \%$ of the reference at the downstream boundary of the CTV.

When the protons have such high energy at the marker position that they do not stop in the marker, they are strongly scattered and create a hot shell surrounding the cold line (see Fig. 7). The maximum dose is then 86 Gy $(\mathrm{RBE})(\phi 2 \mathrm{~mm})$ and $84 \mathrm{~Gy}(\mathrm{RBE})(\phi 1.5 \mathrm{~mm})$ for one field and is reduced to $81 \mathrm{~Gy}(\mathrm{RBE})(\phi 2 \mathrm{~mm})$ and $80 \mathrm{~Gy}$ (RBE) $(\phi 1.5 \mathrm{~mm})$ for two opposite fields.

\section{B. TCP comparison}

In Table 2, values of $\mathrm{R}_{\mathrm{TCP}}^{\mathrm{V}}$ are listed for all conditions. First of all, $\mathrm{R}_{\mathrm{TCP}}^{\mathrm{V}}$ is smallest when $\alpha / \beta$ of $1.5 \mathrm{~Gy}$ (RBE) is used because the TCP curve is steeper for smaller $\alpha / \beta$ and the underdosage more severely affects the TCP reduction (Figure 5). With $\alpha / \beta$ of $1.5 \mathrm{~Gy}(\mathrm{RBE})$, even when $\phi 1.5 \mathrm{~mm}$ marker is used, $\delta \mathrm{TCP}$ can be as large as $36 \%$ if only one field is used. Generally the TCP gets worse as the marker is placed downstream of the CTV because $\mathrm{D}_{\text {min }}$ also decreases. $\mathrm{R}_{\text {TCP }}^{\mathrm{V}}$ even reaches zero when $\phi 2 \mathrm{~mm}$ marker is placed at position (D) and (E). When the marker position is shifted from (D) to (E), the increase of $\mathrm{R}_{\text {TCP }}^{\mathrm{V}}$ can be seen for all $\alpha / \beta$. This is because the overlap of dose shaded volume and CTV decreases and/or the minimum dose $\mathrm{D}_{\min }$ itself increases as the marker shifts to downstream.

The TCP is greatly improved by using two opposing fields. $\delta \mathrm{TCP}$ is less than $3 \%$ for $\phi 2 \mathrm{~mm}$ and less than $0.5 \%$ for $\phi 1.5 \mathrm{~mm}$ marker for any position even when $\alpha \beta$ of $1.5 \mathrm{~Gy}(\mathrm{RBE})$ was used. If three fields are used, $\delta \mathrm{TCP}$ is less than $1 \%$ for any position when the $\phi 2 \mathrm{~mm}$ marker is used while it is less than $0.5 \%$ when the $\phi 1.5 \mathrm{~mm}$ marker is used.

\section{DISCUSSION}

In this research, we have studied the effect of dose distortion in the presence of implanted gold markers on TCP in prostate cancer. A marker of $1.5 \mathrm{~mm}$ diameter does not affect the TCPs with any $\alpha / \beta$ of $1.5,3$, and 10 Gy 
(RBE) when two or more fields are used. On the other hand, if a marker of $2.0 \mathrm{~mm}$ diameter is used, it is safe to use more than two fields especially when multiple markers (two or three) are used in order to suppress the sum of STCP's caused by each marker by less than $3 \%$. It is recommended that $\phi 1.5 \mathrm{~mm}$ markers be used to avoid the reduction of TCP as well as to spare the surrounding critical organs as long as they are visible on fluoroscopy. In addition, it is preferable to place the markers so that they are not close to the distal edge for any of the beams. It is worth mentioning that when multiple markers are used, they have to be placed so that the dose-distorted regions do not overlap with each other. This is achieved by slightly shifting the gantry and/or couch rotation angle by referring to the CT images.

It is clear that the $\mathrm{D}_{\min }$ approaches the reference value (without a marker) by the use of multiple-field irradiation, but it is not a priori obvious whether the increase of fields improves the TCP. Indeed, multiple-field irradiation can mitigate the local dose distortion, but at the same time, the dose shaded region may be expanded. Our results indicate that, as long as $\phi 2 \mathrm{~mm}$ or $\phi 1.5 \mathrm{~mm}$ markers are used, such dose shaded regions are sufficiently small ( $0.6 \%$ of CTV volume at maximum). The improvement of dose distortion by increasing the number of fields surpasses the expanding of the dose shaded region, resulting in an improvement of TCP. The number of fields is however limited by the minimum deliverable amounts for each spot, spacing between spots, and beam size. In our nozzle, if spot spacing is set at $8 \mathrm{~mm}$, which is about 1.6 times the smallest spot size at the Bragg-peak, three fields is the upper limit for SFUD. It is possible that the number of fields can be increased by the use of Intensity Modulation Proton Therapy (IMPT) plans. By IMPT, however, the proton momentum distribution at certain points is highly dependent on the optimization algorithm as well as the CT images of each patient. It is then difficult to figure out the possible size of the dose distortion and resulting TCPs. SFUD may be safer and simpler from this point of view.

In this research, we performed spot weight optimization on a phantom with fiducial markers removed artificially. The dose distribution is then calculated by returning them to the original positions. This procedure for optimization should also be applied in clinical practice because if spot weight optimization is performed in the presence of markers, there might be a large dose error when the patient geometry changes in an occasion when the prostate deforms or the relative position between the prostate and bones changes. In the dose calculation, it may be difficult or even not worthwhile in reality to restore the markers to the CT image. This is because the manual assignment of gold markers in CT images ${ }^{21}$ is demanding since the slice thickness and pixel width of the CT 
images are comparable to the marker size. Also, metal artifact ${ }^{9}$ prevents pinpointing the marker position. Even if the assignment were possible, a high-precision dose calculation algorithm such as Monte Carlo simulation is required to reproduce the dose disturbance, which is time-consuming. Therefore, it may be a reasonable strategy to perform the dose calculation using the CT image with the marker removed, while implementing the methodology so that the unseen dose distortion does not result in significant effects.

The metal artifacts caused by gold markers may be reduced using proposed metal artifact reduction algorithms for helical $\mathrm{CT}^{22}$ or using the image registration technique between helical CT image and cone beam CT image with metal artifact removed ${ }^{23}$. The proton range precision with these metal-artifact-free CTs should be investigated in future.

Gold markers are also used for accurate patient alignment and motion management for other sites such as liver and lung. In such cases, the marker is generally placed outside of tumor, which may mitigate the possibility of severe dose distortion since our results indicate that the more proximal region the marker is placed, the less dose distortion it occurs. Detailed study on this issue will be carried out in the future.

The shape of the gold marker is limited to a sphere in this study. With the spherical symmetry, it has the advantage over other shape of marker in that the precise 3-dimensional real-time tracking is possible even if the markers rotate during the treatment. On the other hand, the commercially available helical gold markers have an advantage that it may be more stable against migration. According to the Monte Carlo simulation ${ }^{7}$, however, it causes more severe dose shadowing than the marker considered in this study. The smallest implantable marker ( $0.75 \mathrm{~mm}$ in diameter and $1 \mathrm{~cm}$ in length) can cause as much as $64.1 \%$ of underdose when two opposite field is used and the markers are parallel to the beam. This may be because the length of the marker is so long that it greatly reduces the proton fluence in downstream. With this size of underdose, the non-negligible size of TCP reduction is inevitable.

In this study, we neglected the effect of inter- and intra- fractionated tumor motions on TCP. Indeed, by using the real-time tracking of gold markers, both inter- and intra- fractional prostate motions can be suppressed within 2 $\mathrm{mm}^{3}$. Therefore, we consider it a good approximation to neglect these motions in this study. Moreover, in ref ${ }^{24}$, it was shown that the effect of inter- and intra- fractional motion on TCP is small $(<2 \%)$ with patient set up using internal markers.

Finally, our study has several limitations. Firstly, the PTV margin that we considered may not be sufficiently 
large to include the proton range uncertainty. However, the dose distortion and TCP values are expected to be improved with the enlargement of the margin since there is a greater possibility that the marker is placed farther from the distal edge. Secondly, the calculated dose perturbation has not been confirmed by measurement. Thirdly, the dose distortion in a water phantom as well as cubic shape of the CTV may simplify the heterogeneous patient anatomy. Finally, fiducial markers composed of other materials that may also be visible on fluoroscopy have not been explored. Additional study of these issues should be undertaken in the future.

\section{CONCLUSIONS}

In this research, we explored the possibility that multiple-field irradiation improves the underdose effect by implanted gold markers on TCP in prostate cancer for spot-scanning proton therapy. Two sizes of spherical gold marker with diameters of $2 \mathrm{~mm}$ and $1.5 \mathrm{~mm}$ were recognized by fluoroscopy when inserted in a pelvic phantom. For both markers, dose distortion is generally more severe when the marker is close to the distal edge of CTV. A gold marker with $1.5 \mathrm{~mm}$ diameter does not affect the TCPs derived from different biological parameters $(\alpha / \beta)$ and at any position in CTV when two or more fields are used. On the other hand, if multiple gold markers of 2.0 mm diameter are used, it is safe to use more than two fields and place the markers at a distance from the distal edge for all the beams in order to suppress the decrease in $\mathrm{TCP}$ from $\mathrm{TCP}_{\mathrm{r}}$ by less than $3 \%$.

\section{Acknowledgements}

This research is supported by the Japan Society for the Promotion of Science (JSPS) through the "Funding Program for World-Leading Innovative R\&D on Science and Technology (FIRST Program)," initiated by the Council for Science and Technology Policy (CSTP). This work was partly supported by Grant-in-Aid for Young Scientists (B) 23791379.

\section{APPENDIX A: VALIDATION OF MONTE CARLO CODE}

The proton range and size of multiple Coulomb scattering in water and gold were validated with NIST database ${ }^{25}$ and analytical approximation formula of Highland ${ }^{26}$, respectively.

\section{(1) Proton Range}

We considered the relevant four energies of $\mathrm{E}=103,132,157$, and $180 \mathrm{MeV}$. They are the minimum and 
maximum energies for lateral and anterior beams, respectively. The range in the present MC system was obtained by simulating $10^{5}$ protons with all physics processes switched off except for ionization ${ }^{26,27}$. In that case, the depth $\mathrm{Z}_{80}$ at which the dose has dropped to $80 \%$ of its maximum value beyond Bragg-peak should equal the range $\mathrm{R}_{0}$ in NIST database ${ }^{26}$. In water the agreement of $\mathrm{z}_{80}$ and $\mathrm{R}_{0}$ is within $1.6 \mathrm{~mm}$ and in gold it is within $0.7 \mathrm{~mm}$, which are both considered to be negligible.

\section{(2) Multiple Coulomb Scattering}

2-1. scattering in a phantom composed of a single material (water, gold)

Again we considered the above four energies and calculated the beam width $\left(\sigma_{M C}\right)$ at depth of $0.5 R_{0}$ and $0.97 R_{0}$ in water ${ }^{26}$. In gold, only $\sigma_{\mathrm{MC}}$ at depth of $0.97 \mathrm{R}_{0}$ was validated since the proton range in gold was too short, and the resultant increment of $\sigma$ is small. The values were compared with those derived from Highland formula $\left(\sigma_{\text {Highland }}\right)$ ${ }^{28,29}$. In water, $\sigma_{\text {Highland }}$ is a little bit greater than $\sigma_{\mathrm{MC}}$, which agrees with the behaviors investigated before in ref ${ }^{30}$. The difference in $\sigma$ increases as the beam passes through the phantom, but is $0.8 \mathrm{~mm}$ at a maximum which is enough small compared with the total beam width. In gold, $\sigma_{\text {Highland }}$ is smaller than $\sigma_{\mathrm{MC}}$ by $0.3 \mathrm{~mm}$ at most, which is also negligible.

\section{2-2. scattering in a phantom composed of water and gold plate}

In order to do a validatation in a setting closer to our system, the gold plate of $2 \mathrm{~mm}$ thickness was placed in a water phantom (Figure 8). Protons of $\mathrm{E}=180$ and $132 \mathrm{MeV}$ were irradiated as shown in the figure. The beam widths $\left(\sigma_{\mathrm{MC}}\right)$ were compared with those derived from Highland formula $\left(\sigma_{\text {Highland }}\right)$ at the peak position of each beam. Their agreeemnt is both within $0.2 \mathrm{~mm}$, which is enough small.

\section{References}

${ }^{1}$ H. Shirato, S. Shimizu, T. Kunieda, K. Kitamura, M.van Herk, K. Kagei, T. Nishioka, S. Hashimoto, K. Fujita, H. Aoyama, K. Tsuchiya, K. Kudo, and K. Miyasaka, "Physical aspects of a real-time tumor-tracking system for gated radiotherapy,” Int. J. Radiat. Oncol. Biol. Phys. 48, 1187-1195 (2000).

${ }^{2}$ D. Litzenberg, J. Balter, S. Hadley, H. Sandler, T. Willoughby, P. Kupelian, and L. Levine, "Influence of intrafraction motion on margins for prostate radiotherapy,” Int. J. Radiat. Oncol. Biol. Phys. 65, 548-553 (2006). 
${ }^{3}$ S. Shimizu, Y. Osaka, N. Shinohara, A. Sazawa, K. Nishioka, R. Suzuki, R. Onimaru, and H. Shirato, "Use of Implanted Markers and Interportal Adjustment with Real-Time Tracking Radiotherapy System to Reduce Intrafraction Prostate Motion,” Int. J. Radiat. Oncol. Biol. Phys. 81, 393-399 (2011) and the references therein.

${ }^{4}$ N. Mendenhall, Z. Li, B. Hoppe, R. Marcus Jr., W. Mendenhall, R. Nichols, C. Morris, C. Williams, J. Costa, and R. Henderson, "Early Outcomes From Three Prospective Trials of Image-Guided Proton Therapy for Prostate Cancer," Int. J. Radiat. Oncol. Biol. Phys. 82, 213-221 (2012).

${ }^{5}$ W. Newhauser, J. Fontenot, N. Koch, L. Dong, A. Lee, Y. Zheng, L. Waters, and R. Mohan, "Monte Carlo simulations of the dosimetric impact of radiopaque fiducial markers for proton radiotherapy of the prostate," Phys. Med. Biol. 52, 2937-2952 (2007).

${ }^{6}$ W. Newhauser, N. Koch, J. Fontenot, S. Rosenthal, S. Gombos, M. Fitzek, and R. Mohan, "Dosimetric impact of tantalum markers used in the treatment of uveal melanoma with proton beam therapy,” Phys. Med. Biol. 52, 3979-3990 (2007).

${ }^{7}$ A. Giebeler, J. Fontenot, P. Balter, G. Ciangaru, R. Zhu, and W. Newhauser, "Dose perturbations from implanted helical gold markers in proton therapy of prostate cancer,” J. Appl. Clin. Med. Phys. 10, 63-70 (2009).

${ }^{8}$ J. Cheung, R. Kudchadker, R. Zhu, A. Lee, and W. Newhauser, "Dose perturbations and image artifacts caused by carbon-coated ceramic and stainless steel fiducials used in proton therapy for prostate cancer,” Phys. Med. Biol. 55, 7135-7147 (2010).

${ }^{9}$ Y. Lim, J. Kwak, D. Kim, D. Shin, M. Yoon, S. Park, J. Kim, S. Ahn, J. Shin, S. Lee, S. Park, H. Pyo, D. Kim, and K. Cho, "Microscopic gold particle-based fiducial markers for proton therapy of prostate cancer," Int. J. Radiat. Oncol. Biol. Phys. 74, 1609-1616 (2009).

${ }^{10}$ M Zhang, L Levinson, S Goyal, N Yue, and X Mo, "On the Use of Multiple Beam Angles to Minimize the Impact of High Density Fiducial Markers in the Prostate Proton Radiotherapy,” Med. Phys. 38, 3649 (2011).

${ }^{11}$ X. Zhu, N. Sahoo, X. Zhang, D. Robertson, H. Li, S. Choi, A. Lee, and M. T. Gillin, "Intensity modulated proton therapy treatment planning using single-field optimization: The impact of monitor unit constraints on plan quality,” Med. Phys. 37, 1210-1219 (2010).

${ }^{12}$ D. Brenner and E. Hall, "Fractionation and protraction for radiotherapy of prostate carcinoma," Int. J. Radiat. Oncol. Biol. Phys. 43, 1095-1101 (1999).

${ }^{13}$ J. Wang, M. Guerrero, and A. Li, "How low is the alpha/beta ratio for prostate cancer? ” Int. J. Radiat. Oncol. Biol. 
Phys. 55, 194-203 (2003).

${ }^{14}$ S. Levegrün, A. Jackson, M. Zelefsky, M. Skwarchuk, E. Venkatraman, W. Schlegel, Z. Fuks, S. Leibel, and C. Ling, "Fitting tumor control probability models to biopsy outcome after three-dimensional conformal radiation therapy of prostate cancer: pitfalls in deducing radiobiologic parameters for tumors from clinical data," Int. J. Radiat. Oncol. Biol. Phys. 51, 1064-1080 (2001).

${ }^{15}$ G. Folger, V. Ivanchenko, and H. P. Wellisch, “The binary cascade, ”Eur. Phys. J. A 21, 407-417 (2004).

${ }^{16}$ K. Nihei, T. Ogino, M. Onozawa, S. Murayama, H. Fuji, M. Murakami, and Y. Hishikawa, "Multi-institutional phase II study of proton beam therapy for organ-confined prostate cancer focusing on the incidence of late rectal toxicities," Int. J. Radiat. Oncol. Biol. Phys. 81, 390-396 (2010).

${ }^{17}$ D. Yan, F. Vicini, J. Wong and A. Martinez, “Adaptive radiation therapy,” Phys. Med. Biol. 42, 123-132 (1997).

${ }^{18}$ J. Slater, C. Rossi, L. Yonemoto, N. Reyes-Molyneaux, D. Bush, J. Antoine, D. Miller, S. Teichman, and J. Slater, “Conformal proton therapy for early-stage prostate cancer,” Urology 53, 978-984 (1999).

${ }^{19}$ A. Niemierko and M. Goitein, "Implementation of a model for estimating tumor control probability for an inhomogeneously irradiated tumor,” Radiother. Oncol. 29, 140-147 (1993).

${ }^{20} \mathrm{~S}$. Webb and A Nahum, "A model for calculating tumour control probability in radiotherapy including the effects of inhomogeneous distributions of dose and clonogenic cell density," Phys. Med. Biol. 38, 653-666 (1993).

${ }^{21}$ O. Jäkel, "Ranges of ions in metals for use in particle treatment planning," Phys. Med. Biol. 51, N173-N177 (2006).

${ }^{22}$ R. Joemai, P. de Bruin, W. Veldkamp, and J. Geleijns, "Metal artifact reduction for CT: development, implementation, and clinical comparison of a generic and a scanner-specific technique, "Med. Phys. 39, 1125-32 (2012).

${ }^{23}$ Y. Zhang, L. Zhang, X. Zhu, A. Lee, M. Chambers, and L Dong, "Reducing metal artifacts in cone-beam CT images by preprocessing projection data,” Int. J. Radiat. Oncol. Biol. Phys. 67, 924-932 (2007).

${ }^{24}$ W. Song, B. Schaly, G. Bauman, J. Battista, and J. Van Dyk, "Evaluation of image-guided radiation therapy (IGRT) technologies and their impact on the outcome of hypofractionated prostate cancer treatments: a radiobiological analysis," Int. J. Radiat. Oncol. Biol. Phys. 64, 289-300 (2006).

${ }^{25}$ M. Berger, J. Coursey, and M. Zucker, "Stopping-power and range tables for proton," http://www.physics.nist.gov/PhysRefData/Star/Text/PSTAR.html (2000).

${ }^{26}$ T. Bortfeld, “An Analytical Approximation of the Bragg Curve for Therapeutic Proton Beams," Phys. Med. Biol. 41, 1331-1339 (1996). 
${ }^{27}$ T. Aso, A. Kimura, S. Tanaka, H. Yoshida, N. Kanematsu, T. Sasaki, and T. Akagi, "Verification of the dose distributions with GEANT4 simulation for proton therapy,” IEEE Trans. 52, 896-902 (2005).

${ }^{28}$ V. Highland, “Practical remarks on multiple scattering,” Nucl. Instr. Meth. 129, $497-499$ (1975).

${ }^{29}$ B. Gottschalk, “'On the scattering power of radiotherapy protons," Med. Phys. 37, 352-67 (2010).

${ }^{30}$ B. Bednarz, G. Chen, B. Han, A. Ding, and X.G. Xu, "Comparison of particle tracking features in Geant4 and MCNPX codes for applications in mapping of proton range uncertainty,” Nucl. Technol. 175, 2-5 (2010). 


\section{Figures captions}

Figure 1. Phantom geometry, coordinate system, and beam directions.

Figure 2. Schematic of nozzle geometry.

Figure 3. Fluoroscopic images of gold markers in pelvic phantom (100 kV, $80 \mathrm{mAs}, 4 \mathrm{msec}(\mathrm{AP}), 120 \mathrm{kV}, 80$ mAs, $4 \mathrm{msec}(\mathrm{LR}))$. The arrows show $\phi 1.5 \mathrm{~mm}$ markers; other dots are $\phi 2 \mathrm{~mm}$ markers. Two coiled markers with $\phi 1.5 \mathrm{~mm}$ are also shown for comparison.

Figure 4. The marker positions investigated. Rectangles show PTV region and its side length is $\mathrm{L}=3.6 \sim 4.8 \mathrm{~cm}$. $\xi=1.5 \mathrm{~cm}, \eta=1.0 \mathrm{~cm}$.

Figure 5. TCP curve for $\alpha / \beta$ values of 1.5, 3, and $10 \mathrm{~Gy}$ (RBE) for homogeneous dose distribution.

Figure 6. Depth dose distribution along the central beam axis for gold markers with $\phi 2 \mathrm{~mm}$ (left) and $\phi 1.5 \mathrm{~mm}$ (right). Three plots for one lateral field with marker positions (A), (C), and (E) in Figure 4 (a) are shown with the reference (without marker). The dose values at the marker positions (arrows) have no effect on TCP evaluation. In the plots, these values are removed and the endpoints are linearly interpolated. Note that the peak-to-plateau ratio is rather high because the profile is taken along the central axis of the pencil beam as well as the source to axis distance is rather small in our nozzle.

Figure 7. Plane dose distribution for one (a), two opposite (b), and three fields (c) along the central beam axis for gold markers with $\phi 2 \mathrm{~mm}$ placed at the IC. Rectangular regions and black dots show the PTV and gold marker, respectively.

Figure 8. Simulation setup for beam widths velifications in a phantom composed of water and gold plate. (a) 180 $\mathrm{MeV}$ and (b) $132 \mathrm{MeV}$ beam enters the water phantom, passes through the gold plate of $2 \mathrm{~mm}$ thickness, and stops at 20 and $11 \mathrm{~cm}$ depth, respectively. 
Table 1. Radiobiological parameters used in this study.

\begin{tabular}{lccc}
\hline \hline$\alpha / \beta(\mathrm{Gy}(\mathrm{RBE}))$ & 1.5 & 3 & 10 \\
$\alpha\left(\mathrm{Gy}(\mathrm{RBE})^{-1}\right)$ & 0.1200 & 0.1675 & 0.2322 \\
$\rho_{\mathrm{c}}\left(\mathrm{cm}^{-3}\right)$ & $1 . \times 10^{6}$ & $1 . \times 10^{6}$ & $1 . \times 10^{6}$ \\
\hline \hline
\end{tabular}

Table 2. Summary of minimum dose in $\mathrm{CTV}_{\text {ave }}\left(\mathrm{D}_{\min }\right)$ and the minimum value of $\mathrm{R}_{\mathrm{TCP}}$ among $\mathrm{CTV}_{\min }, \mathrm{CTV}_{\mathrm{ave}}$, and $\mathrm{CTV}_{\max }$ $\left(\mathrm{R}_{\mathrm{TCP}}^{\mathrm{V}}\right)$ for each $\alpha / \beta$ value and for each marker size, position, and number of fields.

\begin{tabular}{|c|c|c|c|c|c|c|c|c|c|}
\hline \multirow[b]{2}{*}{$\begin{array}{c}\text { Beam } \\
\text { arrangement }\end{array}$} & \multirow[b]{2}{*}{$\begin{array}{c}\text { marker } \\
\text { position } \\
(\mathrm{x}, \mathrm{z})(\mathrm{cm})\end{array}$} & \multicolumn{4}{|c|}{$\phi 2.0 \mathrm{~mm}$} & \multicolumn{4}{|c|}{$\phi 1.5 \mathrm{~mm}$} \\
\hline & & $\mathrm{D}_{\min }$ & $\begin{array}{c}\mathrm{R}_{\mathrm{TCP}}^{\mathrm{V}} \\
(\alpha / \beta=1.5 \\
\mathrm{Gy}(\mathrm{RBE}))\end{array}$ & $\begin{array}{c}\mathrm{R}_{\mathrm{TCP}}^{\mathrm{V}} \\
(\alpha / \beta=3 \\
\mathrm{Gy}(\mathrm{RBE}))\end{array}$ & $\begin{array}{c}\mathrm{R}_{\text {TCP }}^{\mathrm{V}} \\
(\alpha / \beta=10 \\
\mathrm{Gy}(\mathrm{RBE}))\end{array}$ & $\mathrm{D}_{\min }$ & $\begin{array}{c}\mathrm{R}_{\mathrm{TCP}}^{\mathrm{V}} \\
(\alpha / \beta=1.5 \\
\mathrm{Gy}(\mathrm{RBE}))\end{array}$ & $\begin{array}{c}\mathrm{R}_{\mathrm{TCP}}^{\mathrm{V}} \\
(\alpha / \beta=3 \\
\mathrm{Gy}(\mathrm{RBE}))\end{array}$ & $\begin{array}{c}\mathrm{R}_{\text {TCP }}^{\mathrm{V}} \\
(\alpha / \beta=10 \\
\text { Gy (RBE) })\end{array}$ \\
\hline \multirow{5}{*}{$\begin{array}{l}\text { one field } \\
\text { (field } 1 \text { ) }\end{array}$} & $(0,-1.5)$ & 43.1 & 0.45 & 0.70 & 0.89 & 50.0 & 0.93 & 0.97 & 0.99 \\
\hline & $(0,-1.0)$ & 41.3 & 0.35 & 0.63 & 0.86 & 48.8 & 0.92 & 0.96 & 0.99 \\
\hline & $(0,0)$ & 38.9 & 0.10 & 0.36 & 0.73 & 47.6 & 0.89 & 0.95 & 0.98 \\
\hline & $(0,+1.0)$ & 25.8 & 0.00 & 0.00 & 0.18 & 39.4 & 0.64 & 0.82 & 0.94 \\
\hline & $(0,+1.5)$ & 24.1 & 0.00 & 0.01 & 0.23 & 42.4 & 0.87 & 0.94 & 0.98 \\
\hline \multirow{3}{*}{$\begin{array}{l}\text { two opposite field } \\
\text { (field } 1+2 \text { ) }\end{array}$} & $(0,0)$ & 56.6 & 0.98 & 0.99 & 0.99 & 60.6 & 1.00 & 1.00 & 1.00 \\
\hline & $(0,+1.0)$ & 48.9 & 0.97 & 0.98 & 0.99 & 56.4 & 1.00 & 1.00 & 1.00 \\
\hline & $(0,+1.5)$ & 48.9 & 0.98 & 0.99 & 1.00 & 58.2 & 1.00 & 1.00 & 1.00 \\
\hline \multirow{15}{*}{$\begin{array}{l}\text { three fields } \\
\text { (field } 1+2+3 \text { ) }\end{array}$} & $(+1.5,0)$ & 60.7 & 0.99 & 1.00 & 1.00 & 63.4 & 1.00 & 1.00 & 1.00 \\
\hline & $(+1.5,+1.0)$ & 57.3 & 0.99 & 1.00 & 1.00 & 62.4 & 1.00 & 1.00 & 1.00 \\
\hline & $(+1.5,+1.5)$ & 57.2 & 1.00 & 1.00 & 1.00 & 62.7 & 1.00 & 1.00 & 1.00 \\
\hline & $(+1.0,0)$ & 60.8 & 0.99 & 1.00 & 1.00 & 63.5 & 1.00 & 1.00 & 1.00 \\
\hline & $(+1.0,+1.0)$ & 57.0 & 0.99 & 1.00 & 1.00 & 62.1 & 1.00 & 1.00 & 1.00 \\
\hline & $(+1.0,+1.5)$ & 57.1 & 1.00 & 1.00 & 1.00 & 63.0 & 1.00 & 1.00 & 1.00 \\
\hline & $(0,0)$ & 59.4 & 0.99 & 1.00 & 1.00 & 63.1 & 1.00 & 1.00 & 1.00 \\
\hline & $(0,+1.0)$ & 57.2 & 0.99 & 1.00 & 1.00 & 62.2 & 1.00 & 1.00 & 1.00 \\
\hline & $(0,+1.5)$ & 57.1 & 0.99 & 1.00 & 1.00 & 63.0 & 1.00 & 1.00 & 1.00 \\
\hline & $(-1.0,0)$ & 55.0 & 0.99 & 1.00 & 1.00 & 60.2 & 1.00 & 1.00 & 1.00 \\
\hline & $(-1.0,+1.0)$ & 55.3 & 0.99 & 0.99 & 1.00 & 60.9 & 1.00 & 1.00 & 1.00 \\
\hline & $(-1.0,+1.5)$ & 55.1 & 0.99 & 1.00 & 1.00 & 60.2 & 1.00 & 1.00 & 1.00 \\
\hline & $(-1.5,0)$ & 51.4 & 0.99 & 0.99 & 1.00 & 59.1 & 1.00 & 1.00 & 1.00 \\
\hline & $(-1.5,+1.0)$ & 51.5 & 0.99 & 0.99 & 1.00 & 57.9 & 1.00 & 1.00 & 1.00 \\
\hline & $(-1.5,+1.5)$ & 51.5 & 0.99 & 0.99 & 1.00 & 58.3 & 1.00 & 1.00 & 1.00 \\
\hline
\end{tabular}




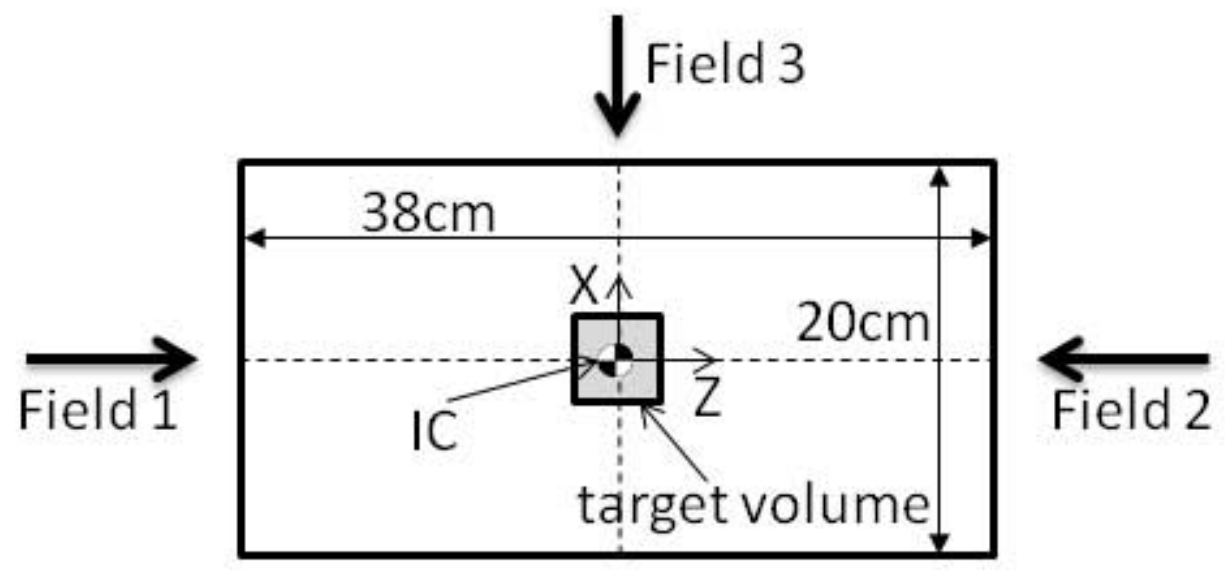


helium chamber

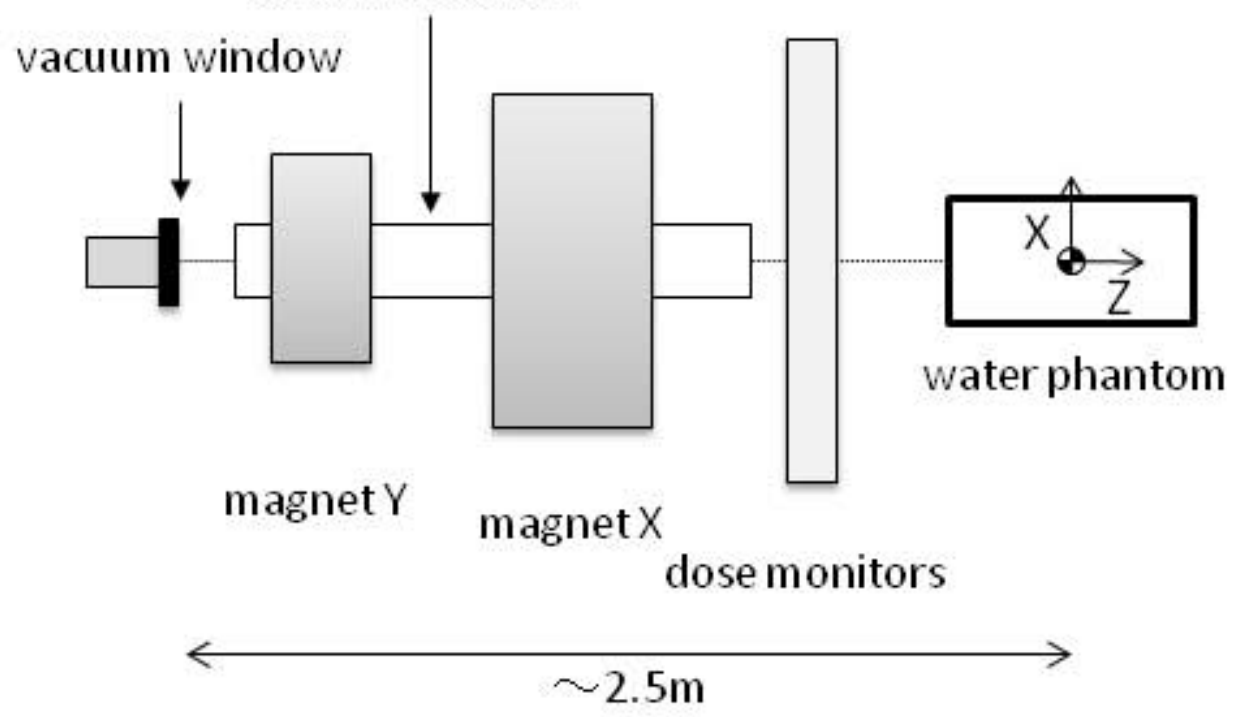



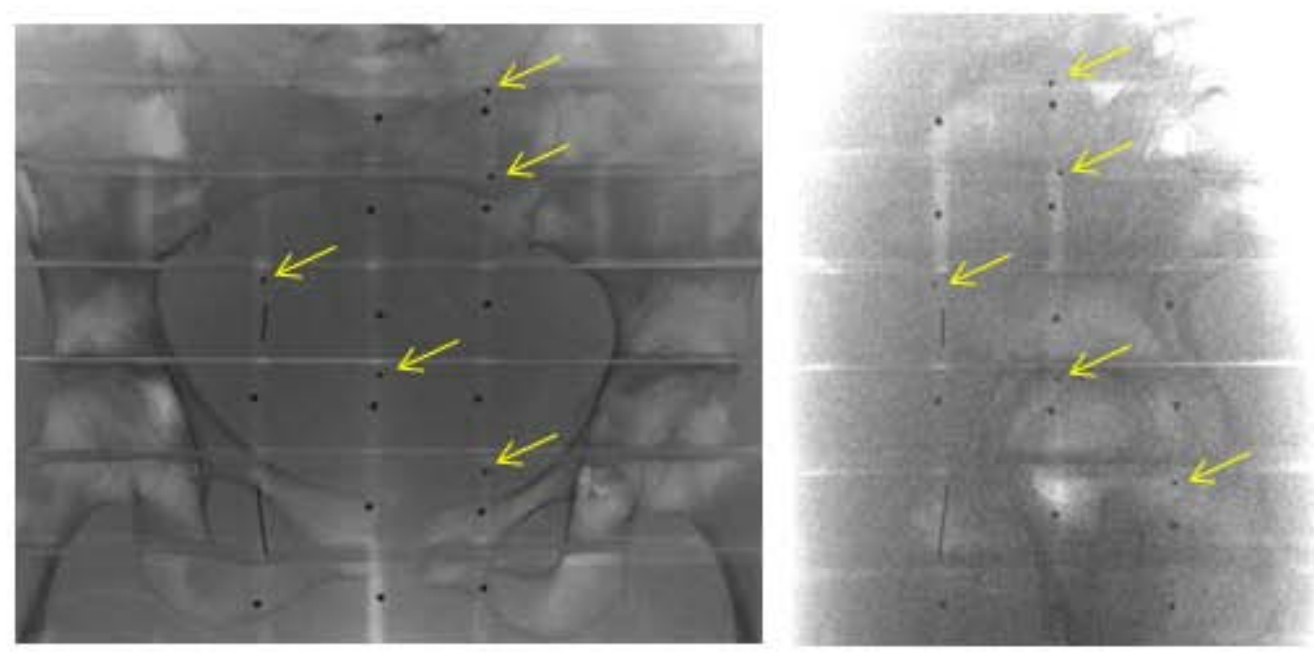


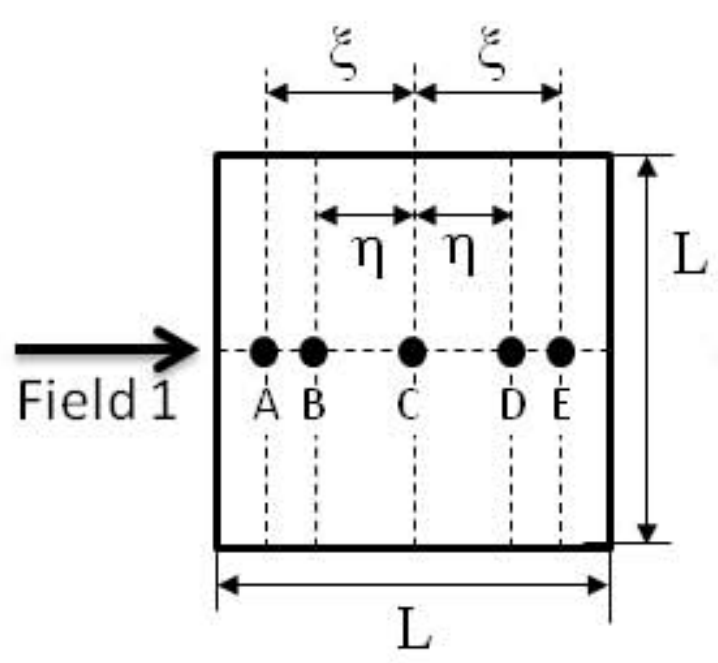

(a) one field

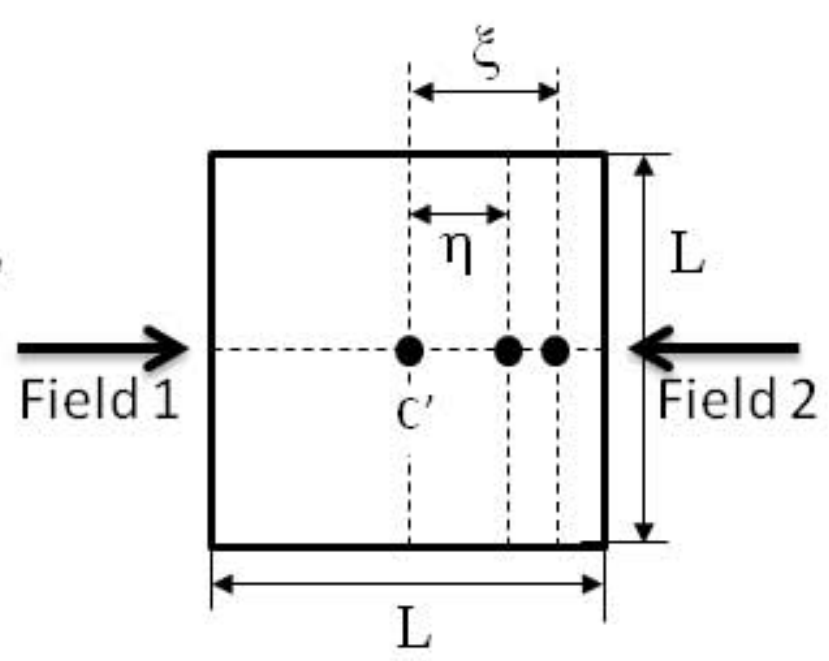

(b) two opposite fields

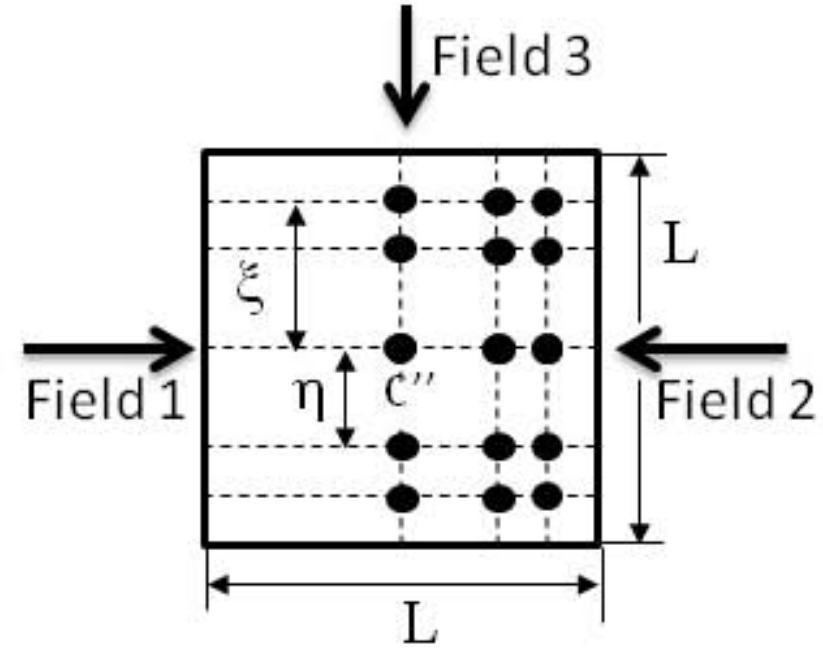

(c) three fields 


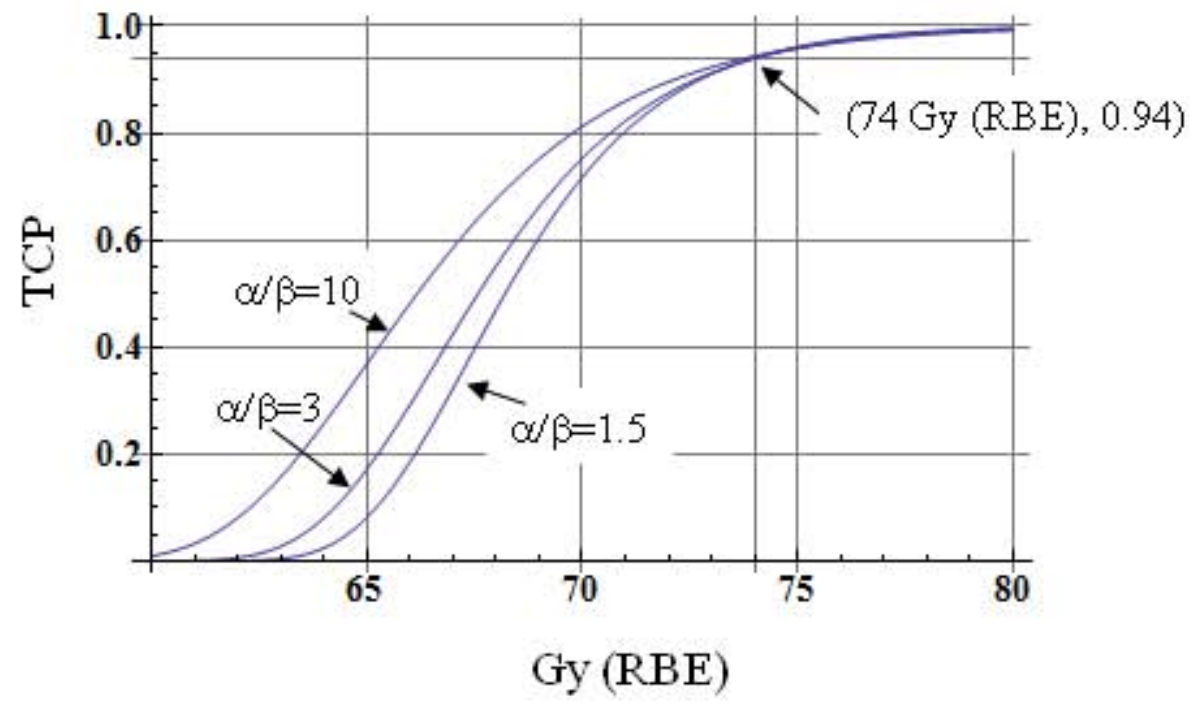




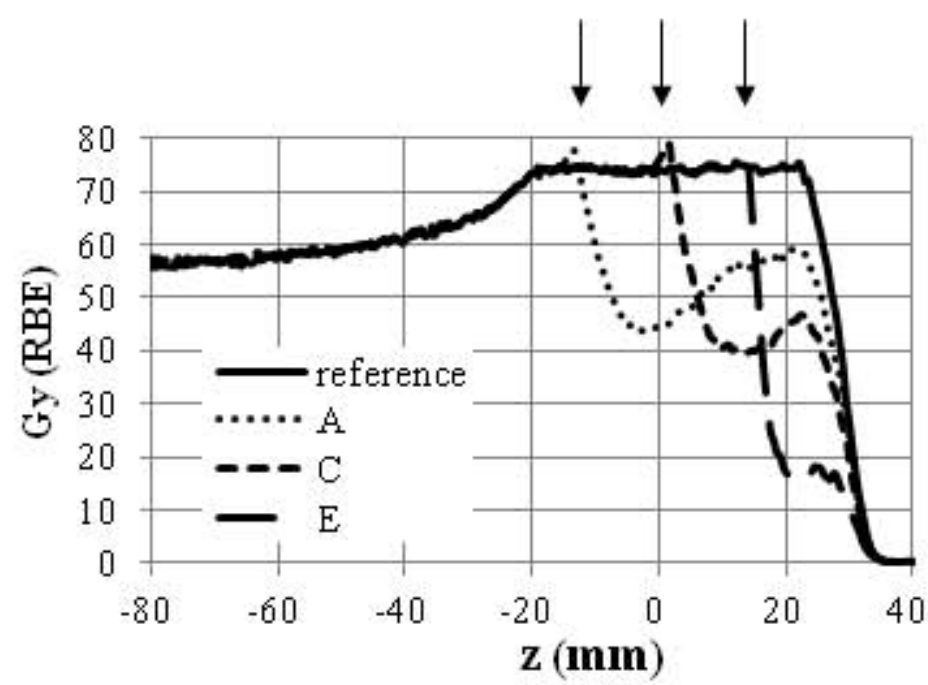

(a)

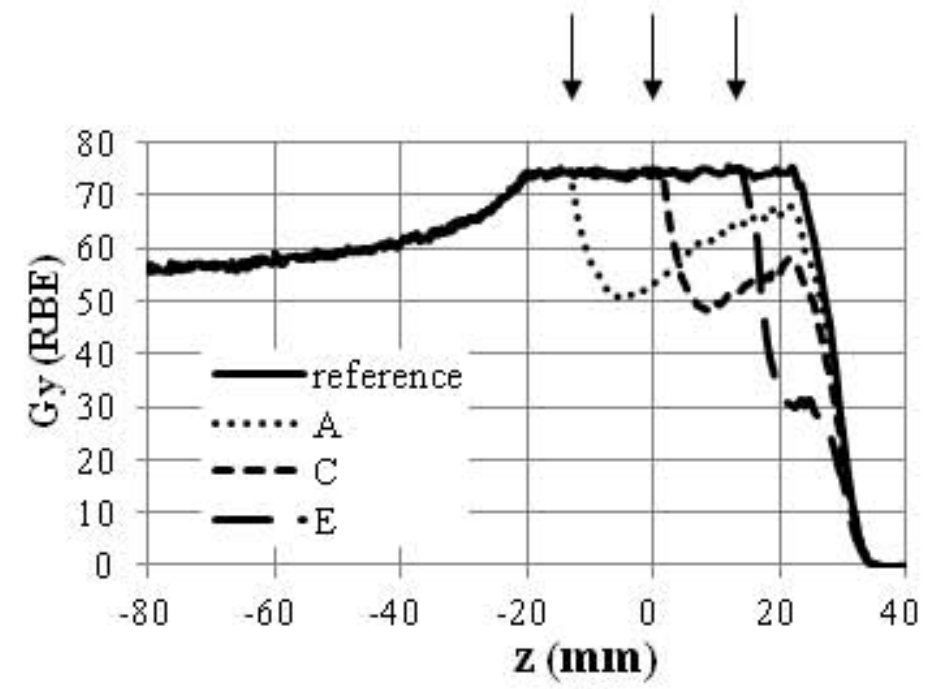

(b) 


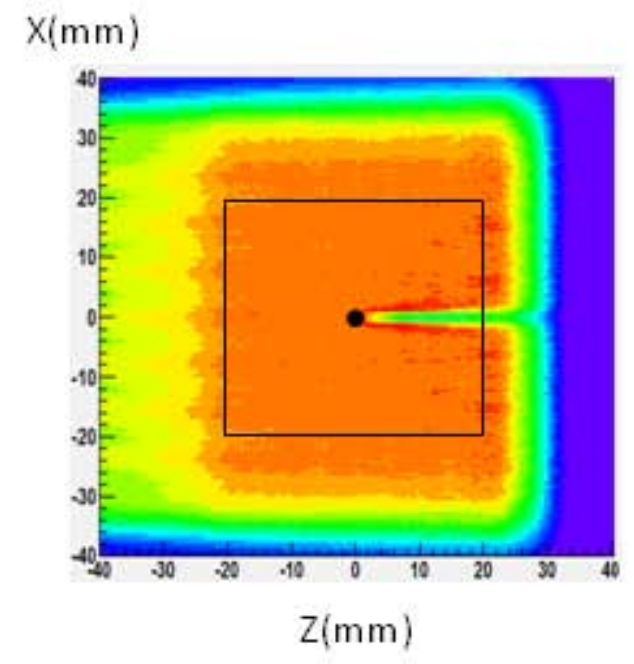

(a) one field

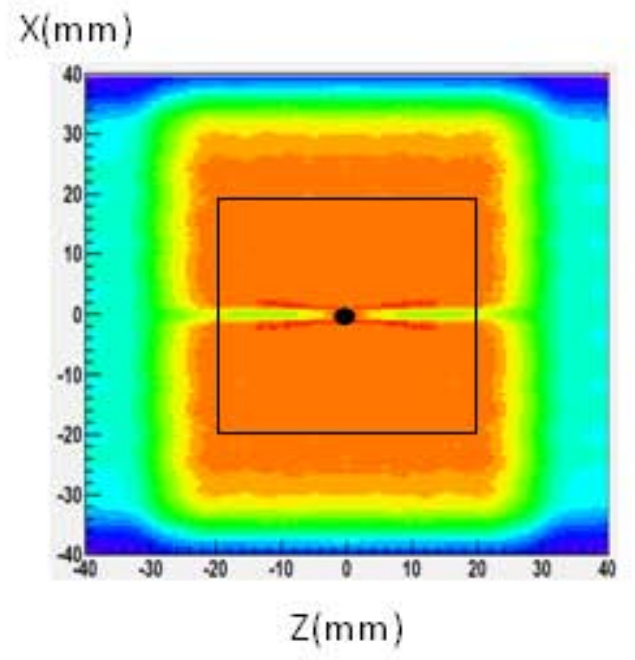

(b) two opposite fields

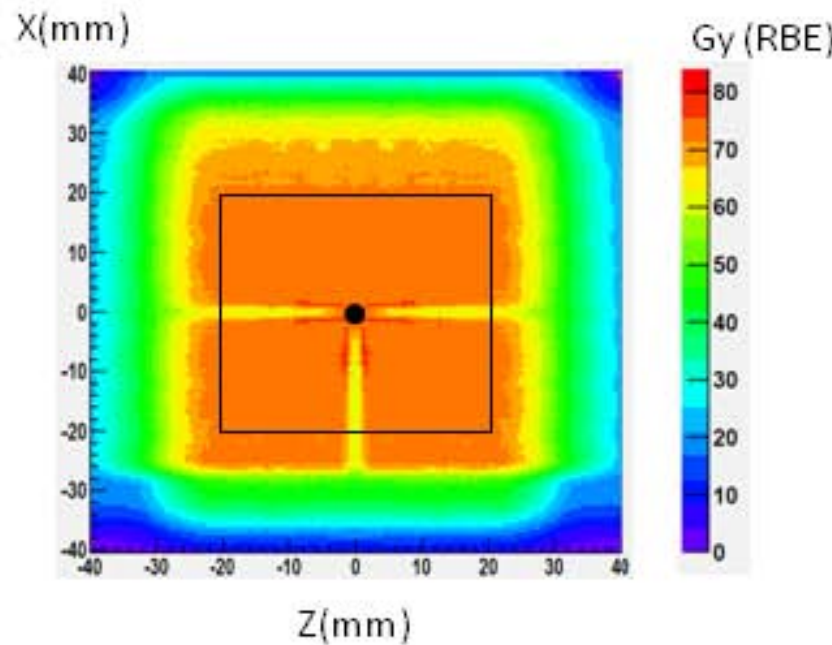

(c) thr ee fields 


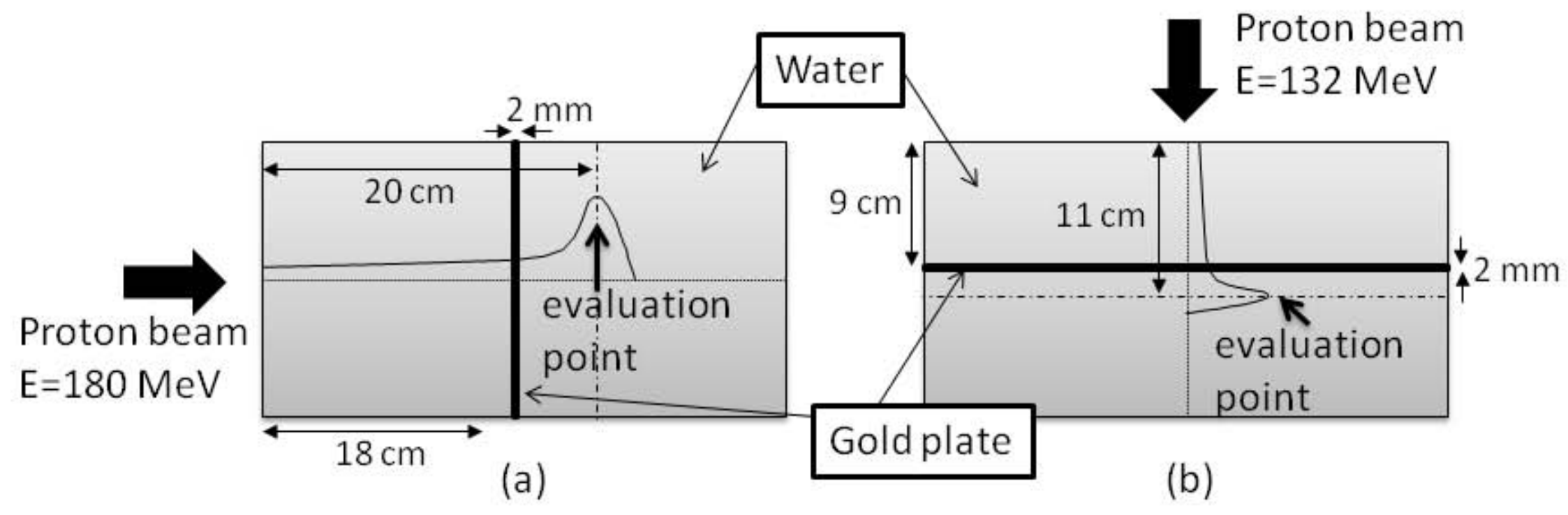

